\title{
GESTÃO DA INOVAÇÃO EM AUDITORIA INTERNA: UMA AVALIAÇÃO DA APLICAÇÃO DA ISO 56.002
}

Ricardo Alexandre Fahl (ricardoafahl@ig.com.br) -Universidade de Araraquara / Programa de Pós-Graduação em Engenharia da Produção

Creusa Sayuri Tahara Amaral (cstamaral@uniara.edu.br) - Universidade de Araraquara / Programa de Pós-Graduação em Engenharia da Produção

\section{RESUMO}

O presente trabalho visa analisar como aprimorar a gestão de inovação de uma auditoria interna, por meio da normatização do processo, utilizando como referência a Norma ISO 56.002 de Sistema de Gestão de Inovação, lançada em 2019. O trabalho parte da premissa de que unidades de Auditoria Interna ocupam posição estratégica relevante nas organizações, agregando conhecimentos aos processos de negócios, que podem ser utilizados tanto no PDP da organização quanto no desenvolvimento de novos processos e métodos para a sua própria atividade. A metodologia do trabalho fundamenta-se na pesquisa bibliográfica, com uma revisão da literatura sobre o tema, para o embasamento técnico e análise das informações. Foram utilizadas publicações em Congressos, revistas especializadas nacionais e internacionais e complementarmente informações de portais de empresas especializadas na atividade de Auditoria Interna. As informações foram analisadas considerando o arcabouço das teorias da inovação e de sua relevância para os processos de negócios. Como resultado verificou-se o importante papel do sistema ISO de inovação, com destaque específico para a ISO 56.002 para o favorecimento ao desenvolvimento de ações estratégicas, de novos processos, de ferramentas e métodos, como melhores práticas e o uso de métricas para a inovação em unidades de auditoria interna.

Palavras chave: Inovação; Auditoria Interna; ISO 56002; Desenvolvimento de Produto 


\section{INTRODUÇÃO}

Conhecimento e inovação são elementos com intrínseca conexão, pois não há inovação sem conhecimento. É a partir do saber que novas ideias são geradas, pois, junto com as competências da organização, o conhecimento promove assimilações que norteiam a inovação (SILVA et al., 2018). As empresas alcançam a vantagem competitiva por meio de iniciativas inovadoras. Essas iniciativas devem ocorrer de forma sistematizada a partir de processos estabelecidos pela organização, denominados processos de inovação (TAJRA, 2020).

O paradoxo sobre a inovação é que todos sabem que ela é fundamental para a sustentabilidade e para o crescimento das empresas, mas poucos conhecem a forma como fazer da inovação o instrumento para a competitividade e poucos possuem a habilidade de gerenciar o processo de inovação (SCHERER, 2016).

Com a percepção sobre a importância da inovação, uma área passou a ganhar maior relevância dentro da empresa: a gestão da inovação. Esta área é responsável por propor, acompanhar e avaliar as práticas de inovação, ou seja, zelar pela manutenção da estrutura necessária para impulsionar a geração de inovação na empresa (CORREA et al., 2020). A Gestão de Inovação visa assim impulsionar o processo ou cultura de inovação sustentável e repetível dentro de uma organização e as iniciativas se concentram em mudanças disruptivas ou graduais (GARTNER, 2021).

A implementação de um sistema de gestão de inovação eficaz e eficiente pode ter impacto ou ser impactado por outro sistema de gestão e pode requerer integração em vários níveis (ASSOCIAÇÃO BRASILEIRA DE NORMAS TÉCNICAS, 2020). A ISO 56.002 foi lançada em julho de 2019, apresentando sua importância, por meio da sistematização de suas diretrizes que podem colaborar com eficiência a gestão de recursos, pessoas e aumentar o desempenho das organizações (BARBOZA et al., 2019).

Considerando esse contexto, o objetivo do artigo é apresentar uma análise de como aprimorar a gestão de inovação da auditoria interna, por meio da normatização do processo, utilizando como referência a Norma ISO 56.002 de Sistema de Gestão de Inovação.

\section{GESTÃO DA INOVAÇÃO}

\subsection{Conceito e contexto}


A inovação pode ser definida como o processo de implementação de novas ideias para criar valor para uma organização, o que pode significar a criação de um novo serviço, sistema, processo, ou o aprimoramento de algum desses elementos (SILVA et al., 2018). Assim, inovações não são fruto de geração espontânea, nem criadas no vácuo, são antes de tudo resultado de intenções deliberadas e geradas num ambiente propício, num terreno fértil, onde as ideias prosperam (SCHERER, 2016).

A inovação é importante para todas as organizações, pois é um requisito para a longevidade. Para colher verdadeiramente os benefícios oferecidos pela inovação, as organizações devem entender que a inovação é um resultado, um processo e uma mentalidade (KAHN, 2018). Desta forma, apresenta-se não como uma atividade eventual, é um processo a ser gerenciado, desde a ideia inicial até a implementação. A inovação deve permear a veia central da empresa, espalhando essa cultura para todo o seu ecossistema (PIERRO, 2020).

A empresa deve conhecer os diferentes modelos de inovação para poder escolher o mais adequado à sua realidade. Em seguida, precisa aproveitar melhor seus recursos (pessoas, infraestrutura, financeiro etc.) para gerenciar seu processo de inovação no dia a dia (BEZERRA, 2011). O processo de inovação pode ser considerado, com base na análise da literatura científica, como um processo organizado e controlado em uma sequência de atividades, em que os insumos na forma de ideias de inovação são transformados em produtos na forma de inovações (LENDEL; HITTIMAR; SIANTOVA, 2015).

Por outro lado, um sistema de gestão da inovação é um conjunto de elementos interrelacionados e interativos, visando a realização de valor. $\mathrm{O}$ sistema fornece uma estrutura comum para desenvolver e implantar recursos para a inovação, avaliar o desempenho e alcançar os resultados pretendidos (ASSOCIAÇÃO BRASILEIRA DE NORMAS TÉCNICAS, 2020).

Todavia, mesmo com a diversidade de ferramentas existentes na literatura e disseminadas pelas consultorias, há muitos problemas na implementação de frameworks para gestão da inovação no contexto real das empresas (BERTAZI; SALERNO, 2015). Os gerentes das empresas conhecem pouco sobre técnicas modernas e ferramentas de design para criar inovação e também sobre a possibilidade de seu uso efetivo para gestão e para o processo de tomada de decisão na área (LENDEL; HITTIMAR; SIANTOVA, 2015).

\subsection{Auditoria Interna}


A Auditoria Interna é uma atividade de avaliação independente e de assessoramento da administração, voltada para o exame e avaliação da adequação, eficiência e eficácia dos sistemas de controle, bem como da qualidade do desempenho das áreas em relação às atribuições e aos planos, metas, objetivos e politicas definidos para as mesmas (INSTITUTO DOS AUDITORES INTERNOS DO BRASIL, 1991). Em termos gerais, a auditoria interna é o processo de examinar, monitorar e analisar as atividades organizacionais para revisar o que a empresa está fazendo para avaliar sua saúde e lucratividade, identificar ameaças potenciais e aconselhar sobre maneiras de mitigar o risco dessas ameaças para minimizar custos (WITTAYAPOOM, 2014).

As áreas de Governança, Risco e Conformidade (GRC) e Inovação tem sido pouco exploradas em pesquisa acadêmicas, e especificamente em pesquisas orientadas para a sustentabilidade (DOYLE et al., 2017). Entretanto, a auditoria interna vem se transformando em uma função que garante um nível adicional de segurança para a gestão da empresa e facilita as condições necessárias para alcançar melhor desempenho global, desenvolvimento sustentável e um ambiente de negócios saudável e competitivo para a empresa (DANESCU; PROZAN; PROZAN, 2015).

A auditoria interna tem papel vital na governança da empresa, que representa uma das formas de relacionamento com a GRC. Ainda, presta avaliação objetiva e oferece conhecimentos sobre a eficácia e a eficiência dos processos de gerenciamento de riscos, de controle interno e de governança (INSTITUTO DOS AUDITORES INTERNOS DO BRASIL, 2018). Os insights da auditoria interna sobre a governança, os riscos e o controle provocam mudanças positivas e inovação dentro da organização.

A auditoria interna constitui um aspecto importante para o programa de inovação de processos, que é crítico para melhorar os programas de desempenho empresarial. A análise de processos, usada no contexto da auditoria interna, concentra-se em programas de melhoria contínua, que se concentram na otimização da combinação e alocação de recursos e estratégias projetadas para minimizar o desperdício e reduzir as atividades sem valor agregado no organização (SISAYE, 1999)

De acordo com os trabalhos de Wong e Cheung (2008) e de Martins e Terblanche (2003) a inovação de auditoria é definida como a capacidade de buscar e criar novas técnicas de auditoria e método diferente para atingir as metas de auditoria e serviço de auditoria (apud HANNIMITKULCHAI; USSAHAWANITCHAKIT, 2016). 
Na organização, a auditoria, quando atuando em processos de inovação, vai além da mensuração, que se baseia em identificar as lacunas entre o desempenho atual e o necessário, podendo identificar onde estão os problemas e as necessidades, fornecer informações que podem ser usadas em planos de ação, para auxiliar no desenvolvimento de melhorias de desempenho (PELLEGRINI et al., 2014)

Embora a atuação da auditoria interna nas empresas seja considerada cada vez mais relevante e necessária, a prática da auditoria interna tem recebido relativamente pouca atenção acadêmica (CHRIST et al., 2020). Assim, sobre o panorama das pesquisas acadêmicas sobre auditoria interna publicadas em periódicos e congressos nacionais, Souza (2017) reportou que a produção acadêmica em auditoria interna é baixa, quando comparada a outras pesquisas bibliométricas na área contábil e constitui um campo que ainda precisa ser melhor explorado pelos pesquisadores.

\section{METODOLOGIA}

Como método de pesquisa para a proposta de análise de como aprimorar a gestão de inovação de uma auditoria interna, por meio da normatização do processo, utilizando como referência a Norma ISO 56.002 foi realizado uma pesquisa bibliográfica. Para o levantamento das informações foi conduzida uma pesquisa exploratória, já que o trabalho cientifico deve iniciar por conhecer todos os aspectos relevantes do tema, de modo a construir um cenário atual, a identificação das lacunas e a proposição de suposições (ANDRADE, 1999). A pesquisa exploratória também é realizada em áreas e sobre problemas em que se há escassez ou nenhum conhecimento acumulado e sistematizado, pela sua natureza, não há hipóteses (DIAS, 2011).

O trabalho utilizou-se de dados de fontes secundárias. As fontes utilizadas foram publicações em Congressos e revistas especializadas nacionais e internacionais, complementadas por informações de portais de empresas de referência e especializadas na atividade de Auditoria Interna. A quantidade de trabalhos encontrados nas buscas estão descritos na tabela 1 .

Após análise do título e resumo, foram selecionados os artigos conforme o foco do estudo. Um conjunto de 17 artigos foram selecionados, analisados e compilados para e desenvolvimento da visão geral sobre o tema e discussão sobre a inovação na auditoria interna com o apoio da norma ISO 56002. 
TABELA 1: Processo de busca

\begin{tabular}{l|l|l}
\hline \hline Base de dados & Palavra-chave & No. de artigos \\
\hline \hline Science Direct & Innovation audit & 161 \\
\hline Science Direct & ISO innovation & 105 \\
\hline Science Direct & Internal audit innovation & 12 \\
\hline ENEGEP ABEPRO & Auditoria interna & 235 \\
\hline ENEGEP ABEPRO & ISO Inovação & 548 \\
\hline CONBREPRO & Auditoria interna & 6 \\
\hline CONBREPRO & ISO Inovação & 31 \\
\hline CONPREBRO & Inovação & 85 \\
\hline \hline
\end{tabular}

Fonte: Dos autores

\section{RESULTADO E DISCUSSÃO}

Para a avaliação das inovações em unidades de auditoria interna mediante as características da ISO 56.002 foi utilizada a ABNT NBR ISO 56.002. Tal documento é uma adoção idêntica, em conteúdo técnico, estrutura e redação à ISO 56.002:2019, que foi elaborada pelo Technical Committee of Innovation Management (ASSOCIAÇÃO BRASILEIRA DE NORMAS TÉCNICAS, 2020). A Associação Brasileira de Normas Técnicas (ABNT) é o Foro Nacional de Normalização.

\subsection{Avaliação ISO 56.002 - Unidades Auditoria Interna}

A capacidade de inovação de uma organização inclui a capacidade de entender e responder às mudanças nas condições de seu contexto, buscar novas oportunidades e alavancar o conhecimento e criatividade das pessoas dentro da organização, e em colaboração com as partes interessadas externas (ASSOCIAÇÃO BRASILEIRA DE NORMAS TÉCNICAS, 2020). A inovação por meio do padrão ISO sugere a criação de um sistema de gestão para facilitar o desenvolvimento da inovação dentro do ambiental organizacional. E este sistema é projetado para funcionar iterativamente, de projeto a projeto, assim, em teoria, o sistema nunca para de funcionar e também evolui continuamente, como um facilitador ao processo do sistema de gestão da inovação das organizações (BARBOZA et al., 2019) 
A ISO 56.002, também conhecida como ISO de inovação, é uma certificação internacional recente, mas que pode se tornar um diferencial para as empresas que desejam incluir e demonstrar ao mercado que possuem uma cultura organizacional voltada para a inovação (ANPEI, 2021). Esta norma é resultado de mais de dez anos de estudos. A ISO de Inovação está direcionada para todas as empresas, independentemente de porte ou segmento.

A Norma foi desenvolvida com as contribuições de uma ampla gama de partes interessadas, incluindo consultores e associações profissionais e, portanto, apresenta a maioria dos elementos que se pode esperar de uma abordagem genérica de alto nível: estratégia, organização, liderança, planejamento, suporte, processo, avaliação de desempenho e melhoria (TIDD, 2021).

O sistema de gestão da inovação, compondo um sistema completo, garante que as empresas criem um modelo baseado na inovação (PIERRO, 2020), entretanto a 56.002 é a única que prevê o atestado de conformidade. O Sistema de Normas ISO Inovação é composto por oito normas quem apresentam a maioria dos elementos que se pode esperar de uma abordagem genérica de alto nível (PIERRO, 2020): direção estratégica, abordagem por processos, realização de valor, liderança com foco no futuro, cultura colaborativa, adaptabilidade e resiliência, gestão de incertezas e gestão de insights. A série de padrões ISO 56000 pode ser ilustrada na figura 1 , a seguir.

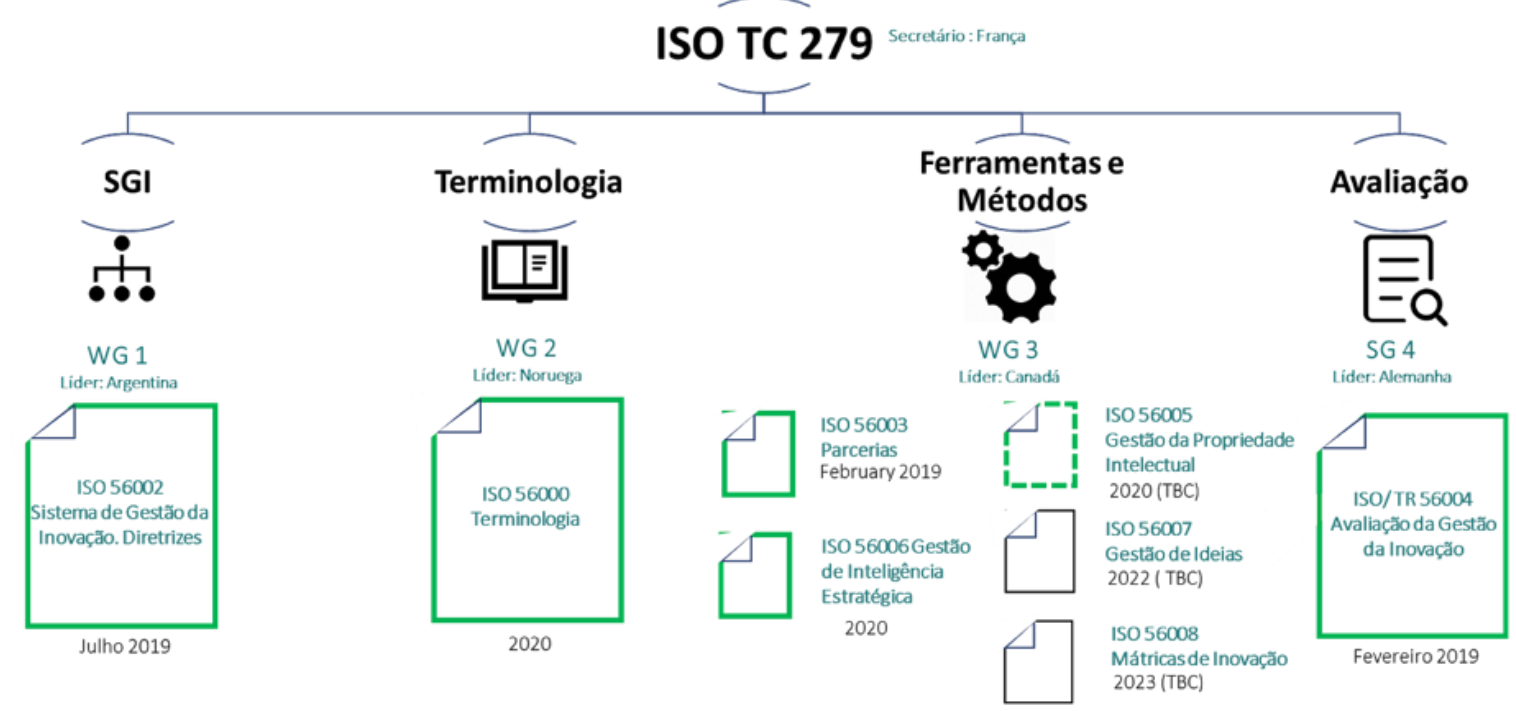

FIGURA 1: O Sistema de Normas ISO Inovação. Fonte: Comparsi (2020).

Um padrão é por natureza uma estrutura genérica de alto nível, mas como resultado, pode falhar em capturar os diferentes contextos das organizações e os desafios dos gerentes, as capacidades da empresa e o tamanho da organização. Assim, torna difícil fornecer um kit 
de ferramentas padrão para uso universal, mas em vez disso, o objetivo deve ser mapear ferramentas úteis para os desafios de gestão específicos e contextos organizacionais diferenciados (TIDD, 2021).

As atividades de inovação precisam lidar com altos graus de variação e incerteza, principalmente durante as fases criativas iniciais. Eles são exploratórios e caracterizados por pesquisa, experimentação e aprendizado. A medida que o processo avança, o conhecimento é adquirido e a incerteza é reduzida (ASSOCIAÇÃO BRASILEIRA DE NORMAS TÉCNICAS, 2020).

Até recentemente, a profissão de auditoria interna não havia enfrentado a necessidade de inovar, e muito menos de se reinventar. Entretanto, sem aplicar novas abordagens, o papel da auditoria interna estaria defasada em relação aos desenvolvimentos estratégico e tecnológico, incapaz de satisfazer as necessidades das partes interessadas e mal equipado para enfrentar riscos emergentes (DELOITTE, 2018).

É evidente que o trabalho de auditoria interna vem se modificando ao longo dos anos e à medida que as empresas crescem e se modernizam com novos sistemas e tecnologias, desenvolvem novos caminhos e controles, também precisam de acompanhamento e atualizações constantes (IMONIANA; MATHEUS, PERERA; 2014). Na era do big data, as funções de auditoria interna devem inovar para incorporar novas técnicas para adicionar valor para suas organizações (RAKIPI; SANTIS; D’ONZA, 2021). Entretanto, muito antes, Sisaye (1999) já apontava que a auditoria interna poderia ser a chave para o processo de inovação nas organizações, considerando que a auditoria não identifica apenas a qualidade dos processos nas organizações, ela também identifica lacunas entre o desempenho atual e o desejado.

Uma unidade de auditoria interna atua para a promoção de uma estrutura organizacional adequada, bem como para a melhoria contínua da eficiência e eficácia para o sistema de liderança da organização, com base na rigorosa gestão de riscos, no controle interno e em processos de governança (DANESCU; PROZAN; PROZAN, 2015). Assim, adotar abordagens inovadoras auxilia para que a função da auditoria mantenha-se atualizada com os novos modelos de desenvolvimentos, posicionando a auditoria interna para antecipar e, em seguida, responder e atender efetivamente às necessidades das partes interessadas, e equipar os próprios auditores internos para abordar os riscos de uma forma eficaz e impactante (DELOITTE, 2018).

De acordo com Pierro (2020), pela análise da norma de Inovação, pode-se verificar que entre os benefícios esperados da implementação da ISO 56.002 estão o envolvimento de 
todas as partes interessadas nos projetos de inovação, a contínua geração de ideias, a criação de uma cultura de inovação, além do desenvolvimento de novos produtos e mercados até então não explorados por uma empresa. A ISO 56.002 é baseado em princípios (pilares) para a inovação. Segundo o documento, os seguintes princípios são a base do sistema de gestão da inovação: realização de valor, líderes focados no futuro, direção estratégica, cultura, exploração de insights, gestão da incerteza, adaptabilidade e abordagem sistêmica. Os princípios podem ser considerados como um conjunto aberto a ser integrado e adaptado dentro da organização (ASSOCIAÇÃO BRASILEIRA DE NORMAS TÉCNICAS, 2020).

O primeiro passo para a implantação do sistema de gestão de inovação é realizar um assessment, que avalia o nível de aderência de uma empresa em relação aos pilares da norma (PIERRO, 2020). Cada empresa tem um nível diferente de gestão de processos de inovação. Portanto, é necessário identificar na primeira etapa o nível atual do processo de inovação, a gestão da empresa, descobrir os pontos fracos e que se faça recomendações para a sua melhoria (LENDEL; HITTIMAR; SIANTOVA, 2015). No passo seguinte inicia-se o processo de implementação (PIERRO, 2020).

Quando o sistema de gestão está implantado, é realizado uma auditoria interna, por um terceiro, a fim de identificar possíveis gaps. Só quando tudo está absolutamente pronto é que uma empresa certificadora ISO faz a auditoria de certificação (PIERRO, 2020). Para gerar valor e fazer com que a inovação realmente se torne algo contínuo e não esporádico, as empresas precisam se comprometer não apenas durante o processo de implementação, mas principalmente depois da certificação, a fim de colher frutos realmente satisfatórios (PIERRO, 2020).

A norma de Gestão ISO 56.002 pode interagir com todo o processo de uma Auditoria Interna, de forma cíclica em um sistema que aprende por meio de métricas a buscar a melhoria contínua, em conformidade com um plano estratégico e o uso de melhores práticas, conforme a figura 2 . 


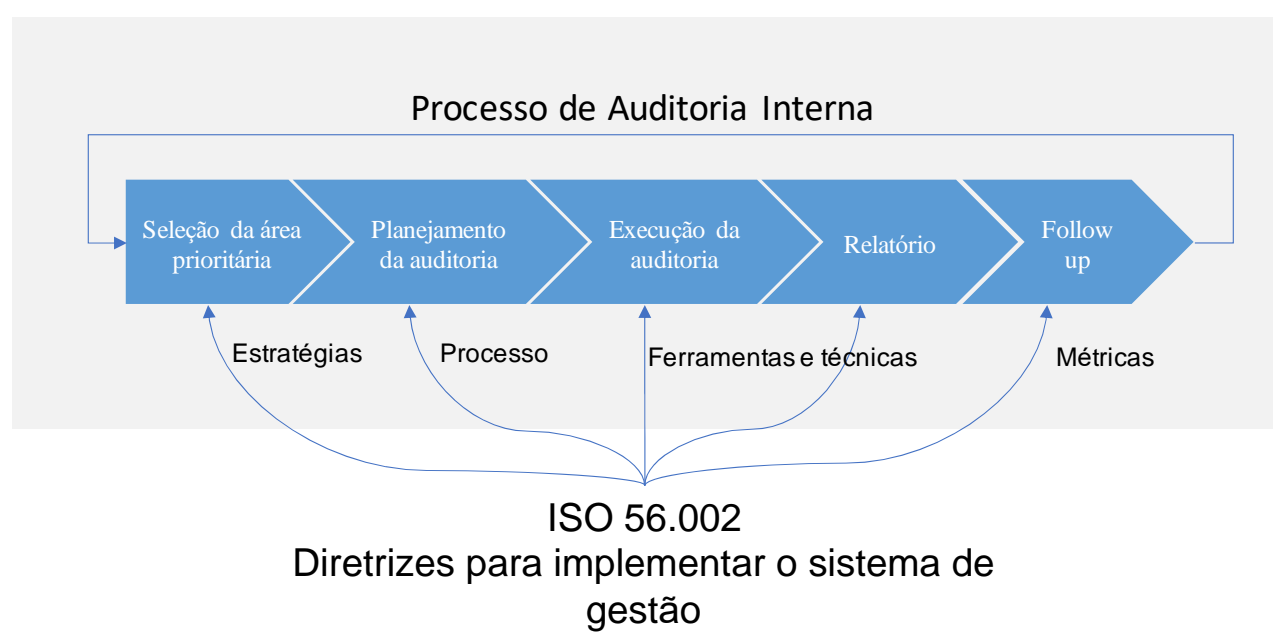

FIGURA 2: Elementos da ISO 56.002 para o aprimoramento do processo de auditoria interna. Fonte: Dos autores.

Como norma de diretrizes, a ISO 56.002 demanda conhecimentos muito mais específicos. É preciso ter um amplo e vasto conhecimento sobre o universo da inovação, sua mentalidade, novas tecnologias e modelos de negócios disruptivos. E por não ser uma norma de requisitos, a implementação tende a ser muito mais subjetiva e complexa. As ações precisam ser testadas até que se chegue às práticas de sucesso específicas de cada empresa (PIERRO, 2020).

\section{CONCLUSÃO}

Este trabalho apresentou uma breve revisão de literatura que permitiu analisar alguns elementos dos modelos de gestão da inovação, para permitir o entendimento do padrão internacional, ISO 56.002 de Sistemas de Gestão da Inovação e identificar sua aderência com uma unidade de auditoria interna.

A inovação por meio do padrão ISO sugere a criação, a partir dos princípios de gestão em inovação, de um sistema de gestão para facilitar o desenvolvimento da inovação dentro do ambiental organizacional. E este sistema é projetado para que a organização melhore de forma contínua, adequando seu processo de inovação de forma regenerativa e gradativo no seu sistema de gestão de inovação, de forma que possa iniciar em um único departamento e expandir-se para toda organização.

O caso de aplicação da ISO 56002 à auditoria interna pode ser verificado como viável e alinhado ao processo. Os conhecimentos iniciais levantado neste trabalho podem contribuir 
para as futuras discussões, já que estamos participando dos estudos preliminares sobre o assunto, que deve ganhar difusão, dado sua importância para o contexto da gestão da inovação nas empresas. Trabalhos futuros sobre casos de implantação, com resultados de sucessos, dificuldades e identificação de necessidade de melhorias devem ser desenvolvidos.

Para a área de auditoria interna, vislumbra-se importante contribuição, dada a importância da área dentro das empresas. Espera-se a partir deste trabalho, desenvolver o aprimoramento dos estudos para identificar como a série de normas ISO 56000 pode contribuir para a gestão da inovação das empresas, ampliando a base de pesquisa da literatura relacionada.

\section{REFERÊNCIAS}

ASSOCIAÇÃO BRASILEIRA DE NORMAS TÉCNICAS. NBR ISO 56002:2020: Gestão da inovação Sistema de gestão da inovação - Diretrizes. Rio de Janeiro. 2020.

ASSOCIAÇÃO NACIONAL DE PESQUISA E DESENVOLVIMENTO DAS EMPRESAS INOVADORAS. ISO de Inovação: Como será certificada a qualidade da gestão de inovação, 2020. Disponível em: https://anpei.org.br/iso-de-inovacao-56002-gestao-da-inovacao. Acesso em 01 de junho de 2021.

ANDRADE, M. A. Introdução à Metodologia do Trabalho Científico. São Paulo: Atlas, 1999.

AUDITORIA INTERNA. Instituto dos Auditores Internos do Brasil, 2018. Disponível em: https://iiabrasil.org.br. Acesso em 01 de maio de 2021.

BARBOZA, B. M. L.; KOVALESKI, J. L.; CHIROLI. D. M. G. ISO 56002: um facilitador de inovação para as organizações. In: CONGRESSO BRASILEIRO DE ENGENHARIA DA PRODUÇÃO, 2019, Ponta Grossa (PR). Anais...Ponta Grossa: CONBREPRO, 2019.

BERTAZI, L. E.A; SALERNO, M. S. Evolução e Panorama da Pesquisa em Indicadores da Inovação. In: SIMPÓSIO DE ENGENHARIA DE PRODUÇÃO,2015, Bauru (SP). Anais...Bauru: SIMPEP, 2015.

BEZERRA, C. A Máquina de Inovação: Mentes e Organização na Luta por Diferenciação. Porto Alegre: Bookman, 2011.

CARVALHO, H. G. Gestão da Inovação. Curitiba: Aymará, 2011.

COMPARSI, L. ISO 56000: uma norma ISO para inovação? Disponível em: https://www.anlab.com.br/2020/11/25/iso-5600/. Acesso em 13 de junho de 2021.

CORREA, R. S.; MIYASHITA, R.; OLIVEIRA, A. R.; PORTELA, L. S. Auditoria de Inovação: Aplicação de Uma Ferramenta de Auditoria Para Avaliar os Processos de Uma Empresa Quanto à Gestão da Inovação. In: SIMPÓSIO DE ENGENHARIA DE PRODUÇÃO,2020, Bauru (SP). Anais...Bauru: SIMPEP, 2020.

CHRIST, M. H.; EULERICH, M.; KRANE, R.; WOOD, D.; A New Frontiers for Internal Audit Research, 2020. Disponível em: https://ssrn.com/abstract=3622148. Acesso em 01 de junho de 2021.

DANESCU, T.; PROZAN, M.; PROZAN, R. D. The Valances of the Internal Audit in Relationship with the Internal Control - Corporate Governance. Procedia Economics and Finance. v. 26, p. 960 - 966, 2015.

DELOITTE. Auditoria Interna 3.0. Disponível em: https://www2.deloitte.com/br/pt/pages/risk/articles/internalaudit-3-0.html. Acesso em 01 de junho de 2021.

DOYLE, E.; MCGOVERN, D.; MCCARTHY, S.; ALANIZ, M. P. Compliance-Innovation: A Quality-Based Route to Sustainability. Journal of Cleaner Production, p. 266 - 275, 2018. 
HANNIMITKULCHAI, K.; USSAHAWANITCHAKIT, P. Continuous Audit Development and Audit Survival: Evidence From Tax Auditors in Thailand. The Business and Management Review, v. 7, p. 487 - 498, 2016

IMONIANA, J. O.; MATHEUS, C. P.; PERERA, L. C. J. Medição de Desempenho de Auditoria Interna: Um Estudo Empírico. Revista Universo Contábil. v. 10, p. 65 - 93.

INNOVATION MANAGEMENT. Gartner Glossary. Disponível em: https://www.gartner.com/en/informationtechnology/glossary/innovation-management. Acesso em: 29/04/2021.

INSTITUTO DOS AUDITORES INTERNOS DO BRASIL. Procedimentos de Auditoria Interna Organização Básica. São Paulo, 1991.

KAHN, K. B. Understanding Innovation. Business Horizons. v. 61, p. 453 - 460, 2018.

LENDEL, V.; HITTMAR, S.; SIANTOVA, E. Management of Innovation Processes in Company. Procedia Economics and Finance, v. 23, p. $861-866,2015$.

PEllegrini, V. L. B.; BARBOSA, J. G. P.; PITASSI, C.; OliVEIRA, M. A. Auditoria de Processos de Inovação: Um Estudo de Caso no Inmetro. Sociedade, Contabilidade e Gestão. v. 9, p. 124 - 141.

PIERRO, A. Normatizando a Inovação. Revista HSM Management, São Paulo, n. 139, p. 22 - 25, abr. 2020.

RAKIPI, R. SANTIS, F. D’ONZA, G. Correlates of The Internal Audit Function's Use of Data Analytics in the Big Data Era: Global Evidence. Journal of International Accounting, Auditing and Taxation. v. 42 , p. 2 39. 2021.

SCHERER, F. O. Gestão da Inovação na Prática: Como Aplicar Conceitos e Ferramentas Para Alavancar a Inovação. São Paulo: Atlas, 2016.

SELVA, C. R. G.; PINTO, L. S.; Auditoria Interna: Colaboradores São Líderes da Inovação. In: Inovação e Políticas Públicas: Superando o Mito das Ideias. Brasília (DF): IPEA, 2019. cap.12, p. $227-240$.

SILVA, F. P.; LIMA, A. P. L.; ALVES, A.; JUNIOR, R. S. C.; DIAS, I.A. M.; DUARTE, M. F. Gestão da Inovação. Porto Alegre: SAGAH, 2018.

SISAYE, S. An organizational approach for the study of the diffusion of process innovation strategies in internal auditing and control systems. International Journal of Applied Quality Management. v. 2, p. 279 - 293 , 1999.

SOUZA, R. G.; Auditoria Interna: Um Panorama das Pesquisas Acadêmicas Publicadas em Periódicos e Congressos Nacionais. Goiânia, 2017. 37 p. Trabalho de Conclusão de Curso (Graduação Ciências Contábeis) - Faculdade de Administração, Ciências Contábeis e Ciências Econômicas.

TIDD. J. A Review and Critical Assessment of the ISO56002 Innovation Management Systems Standard: Evidence and Limitations. International Journal of Innovation Management. 2021.

TJARA, S. F. Inovação na Pratica: Design Thinking e Ferramentas Aplicadas a Startups. Rio de Janeiro: Alta Books, 2020.

TRALDI, M. C.; DIAS, R. Monografia Passo a Passo. São Paulo: Alínea, 2011.

WITTAYAPOOM, K. New Product Development, Accounting Information, and Internal Audits: A Proposed Integrative Framework. Procedia - Social and Behavioral Sciences, v. 148, p. 307 - 314, 2014. 